\title{
BMJ Open Cross-sectional hospital-based study on the seroprevalence of hepatitis B virus markers among healthcare workers, NWR, Cameroon
}

\author{
Etheline W Akazong (D) , ${ }^{1}$ Christopher Tume, ${ }^{1,2}$ Lawrence Ayong, ${ }^{3}$ \\ Richard Njouom (10 , Sebastien Kenmoe, ${ }^{4}$ Ripa Njankouo, ${ }^{4}$ Jules-Roger Kuiate ${ }^{1}$
}

To cite: W Akazong $\mathrm{E}$, Tume C, Ayong L, et al. Crosssectional hospital-based study on the seroprevalence of hepatitis $B$ virus markers among healthcare workers, NWR, Cameroon. BMJ Open 2021;11:e045716. doi:10.1136/ bmjopen-2020-045716

- Prepublication history for this paper is available online. To view these files, please visit the journal online (http://dx.doi org/10.1136/bmjopen-2020045716).

Received 09 0ctober 2020 Revised 25 March 2021 Accepted 16 April 2021

A) Check for updates

(c) Author(s) (or their employer(s)) 2021. Re-use permitted under CC BY-NC. No commercial re-use. See rights and permissions. Published by BMJ.

${ }^{1}$ Department of Biochemistry, Faculty of Science, University of Dschang, Dschang, Cameroon ${ }^{2}$ Department of Biochemistry, University of Bamenda, Bambili, Cameroon

${ }^{3}$ Malaria Unit, Centre Pasteur Cameroun, Yaounde, Cameroon

${ }^{4}$ Department of Virology, Centre Pasteur of Cameroon, Yaounde, Cameroon

Correspondence to

Pr Jules-Roger Kuiate;

jules.kuiate@univ-dschang.org

\section{ABSTRACT}

Background Hepatitis B virus (HBV) infection is a major public health issue worldwide, with about 257 million people reported to be chronic carriers by the WHO fact sheet updated in 2018. HBV can be contracted via direct contact with infected body fluid and infection is almost always asymptomatic. Although healthcare workers (HCWs) are at high risk of HBV infection, little is known about the prevalence of the various HBV markers among HCWs in Cameroon. The present study was taken to evaluate the prevalence of different HBV serological markers among HCWs in the North-West Region of Cameroon.

Methods This cross-sectional hospital-based study was carried out between April and September 2017 during which $395 \mathrm{HCWs}$ were recruited. The serum of the HCWs were tested for the presence of HBV core antibody, hepatitis B surface antibody, hepatitis B e antibody and hepatitis B surface antigen using Monalisa ELISA kits produced by BI0-RAD laboratories. Data were analysed using SPSS V.20.0.

Results Among the 395 participants, 270 (68.4\%) of them were females, 187 (47.3\%) had been exposed to HBV, $145(36.7 \%)$ had resolved the infection, $42(10.6 \%)$ were current HBV carriers, 10 (2.5\%) were infective, $36(9.1 \%)$ were vaccinated and $172(43.5 \%)$ were still susceptible. Exposure to HBV, past infection and susceptibility were significantly associated with age while the rate of vaccination was significantly associated with the job of the HCW in the health facilities.

Conclusion The prevalence of HBV exposure and infection among HCWs obtained in this study was high while the level of vaccination in this at-risk population was low. Adequate steps should be taken to sensitise this population on HBV and the vaccination procedure.

\section{INTRODUCTION}

Hepatitis B virus (HBV) infection is a viral infection that attacks the liver and can cause either an acute and self-resolving, or a chronic disease. ${ }^{1}$ The HBV, made of a partially double-stranded DNA, belongs to the family of Hepadnaviridae. This virus found in both blood and body fluids of infected persons
Strengths and limitations of this study

- Monalisa ULTRA ELISA kits with $100 \%$ sensitivity and specificity of $99.28 \%$ was used to determine hepatitis $B$ virus (HBV) serological markers.

- Stratified sampling technique, which permits estimation of population parameters for groups within population was used for sampling.

- The hepatitis B surface antibody (anti-HBs) titre was not quantified thus the percentage of healthcare workers considered to be vaccinated in this study is higher than the actual percentage with a protective level of anti-HBs.

- Liver biopsy was not done to prove the complete clearance of the virus in resolved infection and serum HBV DNA was not measured since there is no kit available yet to determine this marker.

- The present study covers a cross-section of health professionals, so caution should be taken while generalising the results.

can be transmitted to the mucus membrane or blood stream of non-infected persons. ${ }^{1}$ According to the WHO fact sheet updated in July 2018, an estimated 257 million people are infected with $\mathrm{HBV}$ worldwide and more than 887000 people died in 2015 due to complications of $\mathrm{HBV}$ including cirrhosis and hepatocellular carcinoma. ${ }^{2}$ The level of $\mathrm{HBV}$ varies widely across $\mathrm{WHO}$ regions with the WHO African and WHO Western Pacific Region sharing the greatest burden $(6.1 \%$ and $6.2 \%$ of the population, respectively). In Cameroon, a sub-Saharan African country, $\mathrm{HBV}$ infection is considered hyperendemic with a prevalence rate estimated at $11.5 \% .^{3}$

$\mathrm{HBV}$ infection is preventable with the presence of a vaccine which confers over $96 \%$ protection to recipients. ${ }^{45}$ In Cameroon, the $\mathrm{HBV}$ vaccine (Zilbrix, a DTPw-HBV combination vaccine) was first introduced into the expanded programme on immunisation (EPI) administered to babies at 6 weeks, 10 
weeks and 14 weeks. ${ }^{6}$ The monovalent birth dose implemented in 2017 is limited to babies born of HBV-positive mothers. This vaccine administered during early childhood can only provide some level of protection during early adulthood. ${ }^{5-7}$

Healthcare workers (HCWs) whose job is to care for the sick and injured are often exposed to blood and other body fluids in the course of their work. Consequently, they are at increased risk of infection with blood borne viruses such as HIV, HBV and hepatitis $\mathrm{C}$ virus. ${ }^{8}$ The risk of infection for health workers depends on the prevalence of the disease in the patient population and the nature and the frequency of exposures. ${ }^{9}$ HCWs, when infected, are at risk of transmitting HBV to their patients. ${ }^{9}$ Because of the risk associated with their occupation, WHO recommends that all non-infected HCWs be vaccinated against HBV. ${ }^{8}$

A national survey in Cameroon on the prevalence of HBV among HCWs reported a national seroprevalence of $8.75 \%$ current infection ${ }^{10}$ while a recent study carried out among HCWs in this region reported a prevalence of $10.6 \% .{ }^{11}$ Very little work has been done on the various HBV serological markers (Hepatitis B core antibody (anti-HBc), Hepatitis B surface antibodies (anti-HBs), Hepatitis B e antibody (anti-HBe) and Hepatitis B e antigen $(\mathrm{HBeAg}))$ to evaluate exposure, natural immunity (past or resolved infection), infectivity, vaccination (acquired immunity) and susceptibility. ${ }^{12}{ }^{13}$ In this study, we therefore set out to evaluate the different serological markers associated with $\mathrm{HBV}$ infection (anti-HBc, anti-HBs, Hepatitis B surface antigen (HBsAg) and anti-HBe). These serological markers were used to evaluate the prevalence of exposure, natural immunity, current infection, infectivity, acquired immunity and susceptibility to HBV among HCWs in our setting. Knowledge on these HBV epidemiological features can assist in the development of specific programmes such as vaccination campaigns for susceptible HCWs and guide health policy makers in prioritising and optimising treatment of infected and/or infective HCWs. This in turn can help public health surveillance institutions in our resourcelimited setting to optimise the available resources.

\section{MATERIALS AND METHODS \\ Study design and setting}

This was a cross-sectional hospital-based study conducted between April and September 2017. The study included 22 health facilities in the Bamenda health district (1 regional hospital, 3 CMAs (Centre medical d'arrondissement), 6 mission hospitals, 5 government health centres and 7 private hospitals). Testing stations were set up in the various wards of the health facilities. Over $70 \%$ of HCWs in the various health facilities were recruited for this study.

\section{Case definition}

In this study, exposure was defined as being tested positive for the anti-HBc only, natural immunity (past/resolved infection) was being tested positive for anti-HBc and anti-HBs, current infection was defined as being tested positive for HBsAg, infective subjects were those who were tested positive for HBsAg and negative for anti-HBe, vaccinated subjects were those who were tested positive for anti-HBs only while susceptible (naïve) subjects were those who were negative for all HBV serological markers. Being tested positive implies they were reactive for the marker of interest.

\section{Sample size and justification}

Sample size was determined using the formula proposed by Scott Smith for determining population proportion sample $\operatorname{size}^{14}: \mathrm{X}=\mathrm{Z}$-score $\times \mathrm{SD} \times(1-\mathrm{SD}) / \mathrm{MOE}$. The proportion of HCWs in the NWR was obtained from a registry which published the national proportions of HCWs per region in $2015 .{ }^{15}$ The confidence level was $95 \%$, giving a Z-score of 1.96, a margin of error (MOE) of \pm 5 and an SD of 0.5 . The calculated sample size using this formula was 385 persons.

\section{Sample collection}

All HCWs present in the Bamenda Health District during the study were invited to participate in this study. HCWs who consented to the study were asked to sign a consent form, fill a self-administered questionnaire after which $4 \mathrm{~mL}$ of blood was collected from them into a red cap (dry) tube. Identification number was used to link participant's laboratory results and the questionnaire. A standardised questionnaire designed by the researcher was used to collect sociodemographic data and HCWs category. HCWs included medical doctors, nurses, laboratory technicians, dentist, pharmacist and hospital auxiliary staff (cleaners, carriers, launders).

\section{HBV serology}

Monalisa ELISA kits produced by BIO-RAD laboratories with sensitivity and specificity greater than $99 \%$ were used to qualitatively determine the different HBV serological markers. Monolisa HBsAg ULTRA ELISA kit was used to test for the presence of HBsAg, Monolisa Anti-HBs PLUS ELISA kit (BIORAD, Marnes- La-Coquette-France) was used to test for the presence of anti-HBs, Monolisa Anti-HBc PLUS ELISA kit was used to test for the presence of anti-HBc while Monolisa HBe Ag-Ab PLUS ELISA kit (BIORAD, Marnes- La-Coquette-France) was used to test for the presence of anti-HBe.

\section{Statistical analysis}

Statistical analysis was performed using the Statistical software IBM SPSS Statistics V.22.0 for mac. Continuous data were expressed as median values with first and third IQRs. Categorical data were expressed as percentages. Pearson's $\chi^{2}(p<0.05)$ was used to assess the significance among study variables. OR was calculated using binary logistic regression.

\section{RESULTS}

\section{Characteristics of study population}

A total of 395 HCWs from the different hospitals in this region participated in the study. Among these, 68.4\% 
Table 1 Baseline characteristics of the study population

\begin{tabular}{lcc}
\hline Variables & Frequency (395) & Percentage (100\%) \\
\hline Sex & & \\
Male & 125 & 31.6 \\
\hline Female & 270 & 68.4 \\
\hline Age intervals (years) & \\
\hline $16-25$ & 166 & 42.0 \\
\hline $26-35$ & 155 & 39.2 \\
\hline $36-45$ & 50 & 12.7 \\
\hline $46-65$ & 24 & 6.1 \\
\hline Job & & \\
\hline Nurses & 224 & 56.7 \\
\hline Lab technicians & 90 & 22.8 \\
\hline Medical doctors & 17 & 4.3 \\
\hline Dentist & 15 & 3.8 \\
\hline Pharmacist & 8 & 2.0 \\
\hline Auxiliary staff & 41 & 10.4 \\
\hline
\end{tabular}

$\mathrm{n}$, frequency.

$(n=270)$ were women (table 1$)$. The $16-25$ years age group represented $42 \%(n=166)$ of the study population. The median age of the study population was 27 years (IQR, 23-32 years). Nurses were the most represented in the HCWs category $(n=224,56.3 \%)$.

\section{Exposure to HBV}

Anti-HBc was used to determine exposure to $\mathrm{HBV}$ (table 2). Of the 395 HCWs who participated in this study, 187 (47.3\%) were tested positive for anti-HBc. A statistically significant association was observed between exposure and age ( $p$ value $<0.001$ ). Exposure to $\mathrm{HBV}$ significantly increased with age and HCWs belonging to the 46-65 years age group had a probability greater than 3.5 times of being exposed to $\mathrm{HBV}$ when compared with those belonging to the 16-25 years age group. There was no significant association between sex, HCWs category and exposure. However, exposure was relatively lower among dentist $(n=4,26.7 \%)$ than among other HCWs. Prevalence of exposure was similar between sexes.

\section{Natural immunity against HBV (HBV clearance)}

A combination of anti-HBc and anti-HBs was used to evaluate natural immunity against HBV (past/resolved infection) (table 2). One hundred and forty-five $(77.5 \%)$ HCWs who had come in contact with HBV had effectively cleared the virus. Natural immunity was significantly associated with age $(p$ value $<0.05)$, and was highest in the $36-45$ years age group $(n=27,54.0 \%)$. HCWs belonging to the $36-45$ years age group showed a three times significantly greater probability of resolving the infection when compared with HCWs belonging to the 16-25 years age group. There was no significant correlation between ability to clear the HBV, sex and HCWs category.

\section{Current HBV infection}

The presence of HBsAg was used to determine current HBV infection (table 3). HBsAg was detected in 42 of the 395 HCWs $(10.6 \%)$. There was no statistically significant association between sex, age, HCWs category and current infection. HBsAg infection was higher among females $(\mathrm{n}=33,12.2 \% ; 1.795,95 \% \mathrm{CI}(0.831$ to 3.875$))$ than among males $(n=9,7.2 \%)$. Majority of HBsAg infected HCWs belonged to the 46-65 years age group $(n=4$, $16.7 \%)$ and were dentist $(n=2,13.3 \%)$.

\section{HBV infectivity among HCWs}

The presence of HBsAg and the absence of anti-HBe were used to evaluate $\mathrm{HBV}$ infectivity (table 3). Among the $10.4 \%$ of HCWs infected with HBV, $23.8 \% \quad(n=10)$ of them and $2.5 \%$ of all the HCWs in this study were infective. There was no significant association between sex, age, job and being HBV infective. More females were infective $(n=8,3.0 \%)$ compared with males $(n=2,1.6 \%)$. The 46-65 years age group recorded the highest prevalence of infective HCWs $(n=2,8.3 \%)$ and were pharmacist $(n=1,12.5 \%)$.

\section{Acquired immunity (vaccinated) HCWs}

The absence of anti-HBc and the presence of anti-HBs were used to determine vaccinated HCWs (table 2). Among the $208 \mathrm{HCW}$ sho had never been exposed to HBV $17.3 \%(n=36)$ of them and $9.1 \%$ of the 395 study participants were vaccinated. There was a statistically significant association between being vaccinated and HCWs category ( $p$ value $<0.05)$. Nurses $(n=15,6.7 \%$; $0.197,95 \%$ CI $(0.056$ to 0.695$)$, p value $=0.012)$ and auxiliary staff $(2,4.9 \% ; 0.141,95 \%$ CI $(0.023$ to 0.874$)$, $\mathrm{p}$ value $=0.036$ ) had a significantly lower probability of being vaccinated when compared with dentist. Males and females had a similar prevalence of acquired immunity (12 (9.6\%) males and $24(8.9 \%)$ females). Most of those vaccinated belonged to the 26-35 years age group $(n=15,9.7 \%)$ and were dentist $(n=4,26.7 \%)$. There was no significant association between acquired immunity, sex and job.

\section{Susceptible HCWs}

Susceptibility was determined by the absence of anti-HBc, anti-HBs and HBsAg in serum (table 3). Among the 395 HCWs who participated in this study, $43.5 \%(n=172)$ of them were still susceptible to HBV. A statistically significant association was observed between age and susceptibility ( $p$ value <0.001). Susceptibility significantly decreased with age and was highest in the 16-25 years age group $(n=90,54.2 \%)$. There was no significant association between susceptibility, sex and HCWs category.

\section{DISCUSSION}

$\mathrm{HBV}$ is a major cause of chronic hepatitis, liver cirrhosis and hepatocellular carcinoma. As a viral infection, which can be transmitted via percutaneous and mucosal 


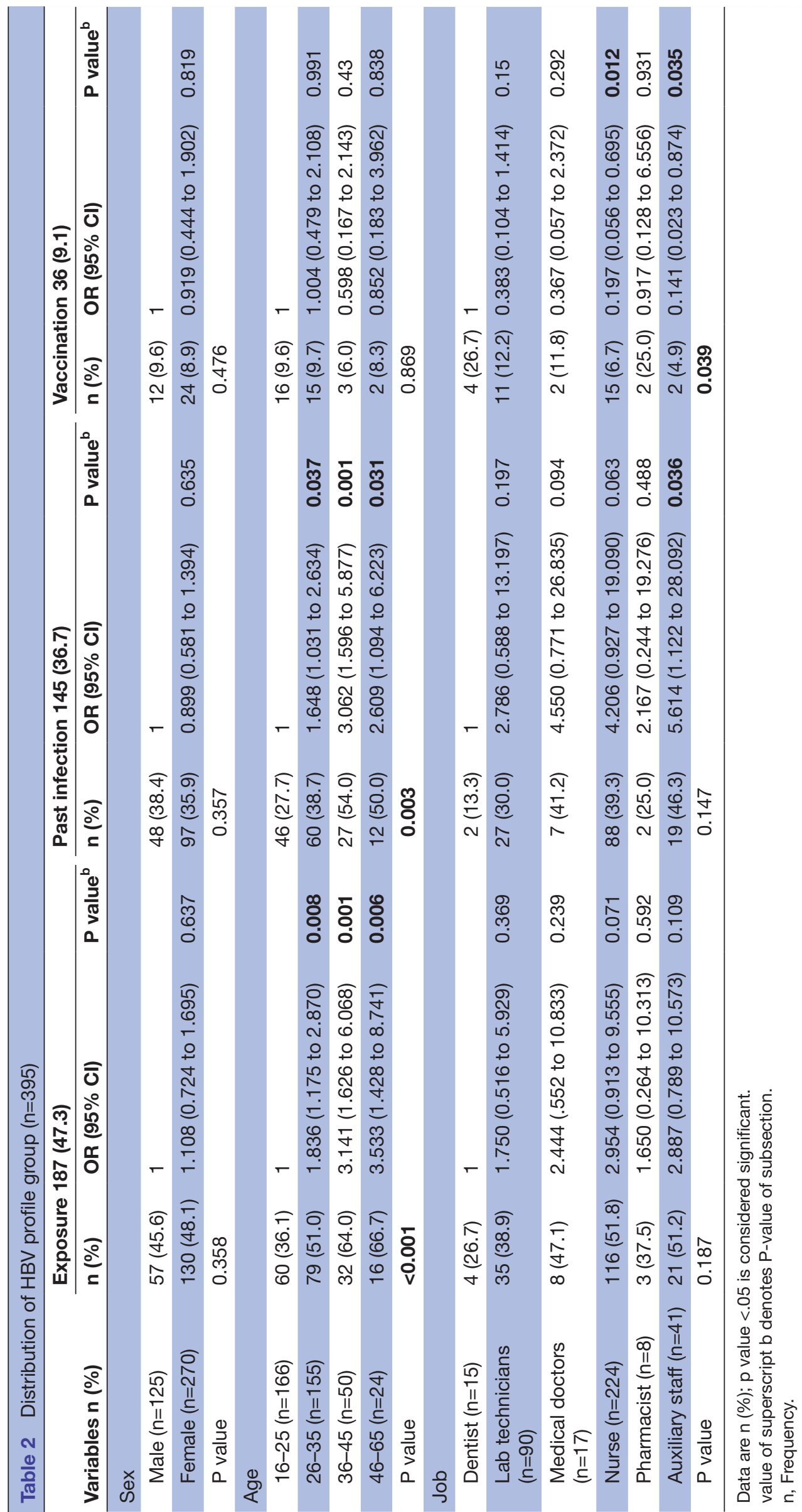




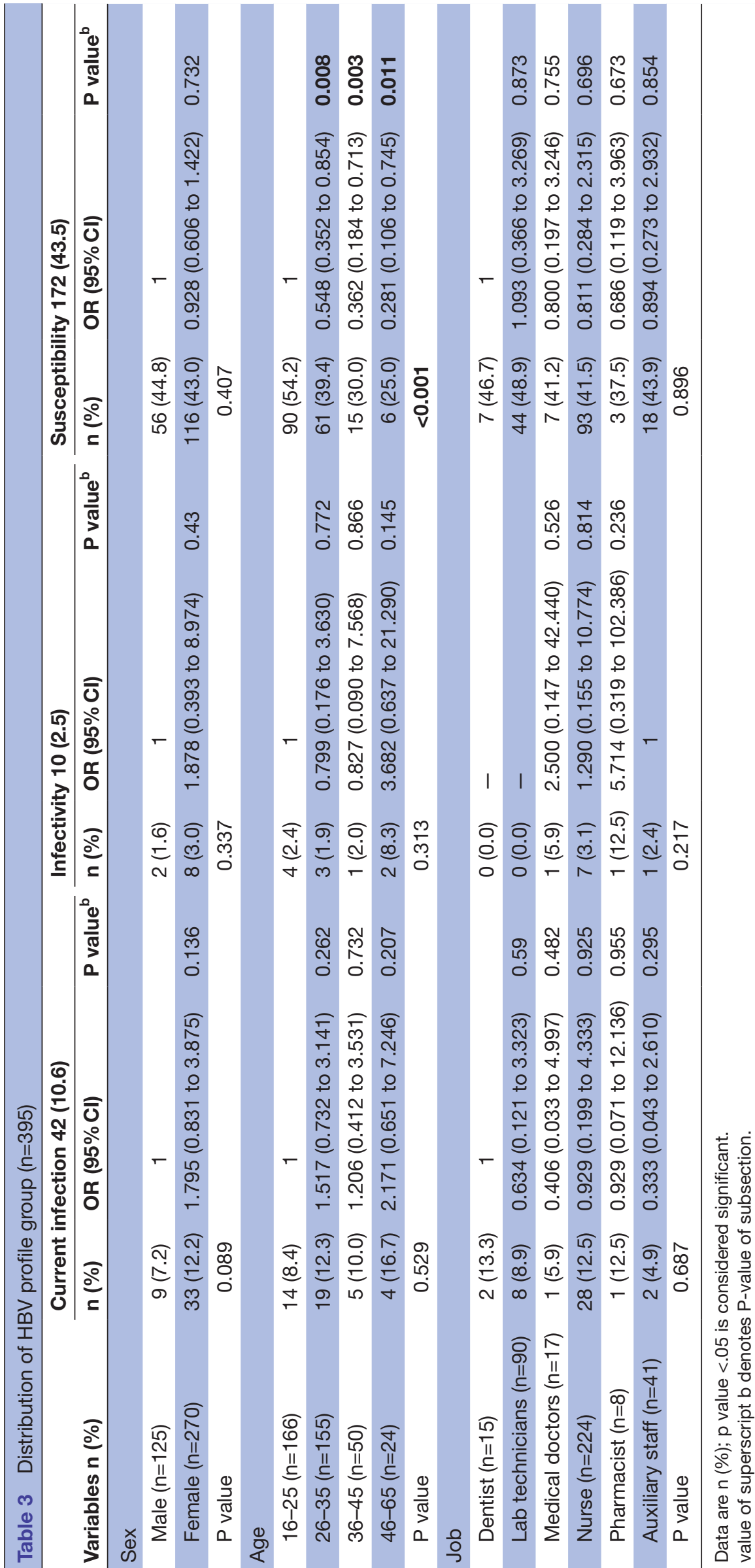


exposure to infective body fluids, HBV stands as a serious nosocomial infection in healthcare settings. The current study aimed at evaluating the seroprevalence of the different HBV serological profiles among HCWs in the NWR of Cameroon showed a high HBV burden in this population. A number of epidemiological and crosssectional studies have reported marked variation in the prevalence of the various HBV serological profiles among HCWs within and out of the country. ${ }^{10-13}{ }^{16-18}$ HBV prevalence in this at-risk group seems to vary with the HBV prevalence in the general population.

\section{Exposure to HBV}

The prevalence of HBV exposure obtained in the current study was high $(47.3 \%)$ and significantly associated with age. This rate of exposure is relatively higher than the $19 \%$ obtained by Tatsilong et al in Yaoundé (the capital of Cameroon) in 2016. ${ }^{12}$ The difference in prevalence could be because of the difference in the diagnostic technique used, given that Tatsilong et al worked with the one-step, rapid strip test which has a relatively lower sensitivity and specificity when compared with the ELISA technique used in this study. ${ }^{19-22}$ Besides, the distribution of the $\mathrm{HBV}$ vaccine in the expanded programme on immunisation (EPI) administered to babies was first introduced in Yaoundé and subsequently to other regions. ${ }^{6}$ This HBV childhood vaccine has been proposed to provide some level of protection against HBV during early adulthood (protection which wanes as you grow older) and might justify the increase in the rate of HBV exposure with age. ${ }^{5-723}$ Finally, older HCWs have spent a longer time in the hospital compared with the younger HCWs most of who are starting in the field.

\section{Natural immunity against HBV (HBV clearance)}

The prevalence of natural immunity among HCWs exposed to HBV was high and was significantly associated with age. In effect, it is known that the clinical course and outcome of HBV infection is greatly influenced by the age at infection, the level of HBV replication and the host immune status. ${ }^{24}$ This might justify the relatively low level of resolved infection in the 16-25 years age group $(27.5 \%)$ which increased up to the $36-45$ years age group $(54.0 \%)$ and finally dropped in the 46-65 years age group $(50 \%)$. However, the prevalence of natural immunity was comparable between the sexes eventhough males had a lower probability of resolving the infection when compared with females. The similarity in prevalence of natural immunity is contrary to what is anticipated given that women generally show a stronger innate and adaptive (humoral and cellular) immune responses when compared with males. ${ }^{25}$ This similarity in prevalence of natural immunity may be justified by the fact that in countries with high prevalence of HBV, exposure to HBV often occurs during birth and early childhood and infection may progress for $20-25$ years in a subtle manner as stated above. The EPI evoked earlier might have reduced the level of exposure to HBV during childhood justifying the seemingly high prevalence of HBV natural immunity among those exposed to HBV. The reason why this disease is self-limiting in some people and not in others have not yet been fully understood. However, it is believed that the host's immune system and the genome of the infecting HBV might play an important role in determining the outcome of the disease in healthy adults.

\section{Current HBV infection}

The prevalence of HBV infection obtained in this study was high $(10.6 \%)$ given that they exist a safe and competent vaccine. The prevalence of $\mathrm{HBsAg}$ positivity is higher than the $8.75 \%$ obtained by Bilounga Ndongo et $a l$ in the NWR in their national survey among HCWs in Cameroon. ${ }^{10}$ The difference in prevalence could be justified by the fact that the national survey focused on the regional hospital (which represents the government reference hospital in this region) and used a different technique (rapid strip test). The same study mentioned above reported a prevalence of 5.4\% in Yaoundé and 24\% in the Far North Region, while Loriette $e t$ al, working in the Far North Region of Cameroon recorded a prevalence of $18 \%$ in $2015 .^{10} 18$ This alternating prevalence could be a reflection of the cultural and climatic differences existing between the different ethnic groups alongside the diverse geographical scenery of the country. ${ }^{10}$

\section{HBV infectivity among HCWs}

HBeAg is a serological marker that indicates the presence of HBV DNA in blood circulation in wild-type HBV. As the immune system clears HBV DNA, HBeAg reduces in the blood circulation as anti-HBe appears. ${ }^{26} 27$ Mutations in some cases can result in HBV DNA being present in blood circulation in the absence of HBeAg. ${ }^{2627}$ However, because there is no ELISA kit to determine the presence of HBV DNA in serum, we defined infectivity as the presence of HBsAg and absence of anti-HBe. This classification of infectivity is the best classification using the ELISA kits but is a limitation to the study given that some infected HCWs can go undetected. Among the $10.4 \%$ of HCWs infected with HBV, $23.8 \%$ of them were infective. The persistence of HBeAg in blood is always associated with progress towards a liver disease as well as an increase probability of transmitting the virus. Even though there exist a management guide proposed by WHO in 2015 , this high prevalence of infectivity among infected HCWs might be because of the elevated cost involved in managing the disease. ${ }^{9} 8$

\section{Acquired immunity (vaccinated) HCWs}

According to the 'CDC updates HBV vaccination guidelines for HCWs' (2014), healthcare personnel should be vaccinated against $\mathrm{HBV}$ before exposure to blood or body fluids and should receive serological testing to assess for anti-HBs. ${ }^{29}$ Still, just $9.1 \%$ of HCWs in our setting showed acquired immunity. HCWs belonging to the 16-25 years age group were the most vaccinated. This might be because of the expanded immunisation 
invoked earlier. ${ }^{6723}$ But most probable is the fact that some institutions now ask for proof of $\mathrm{HBV}$ vaccination in none-infected individuals before hire or matriculation. Overall, the low prevalence of HCWs vaccinated against HBV might either be due to inappropriate sensitisation on $\mathrm{HBV}$ or the cost of the HBV vaccine.

\section{Susceptible HCWs}

HBV susceptibility in our study was high $(43.5 \%)$ and was inversely proportional to age. The statistically significant association between age and susceptibility to HBV might be explained by the decrease in childhood and maternal transmission of HBV due to the expanded immunisation explained above. ${ }^{6}{ }^{23}$ Unfortunately, this infant vaccine cannot provide adequate protection in adulthood and most parents never go for the booster dose because of the cost of the vaccine reducing childhood HBV transmission but increasing the number of susceptible adult HCWs. More so, duration in the hospital increases with age. Thus, the younger HCWs whose duration in healthcare settings is less than that of older HCWs and who still have a longer period to work in this setting have not been exposed to HBV nosocomial risk factors like older HCWs and have a higher risk of eventually getting exposed when compared with older HCWs.

\section{CONCLUSION}

This study revealed a considerable burden (10.6\% current infection) of HBV infection in the Bamenda health District, North West Region of Cameroon. Among the infected HCWs, over $23.8 \%$ of them were infective. These infective HCWs are at risk of infecting their patients. Subsidising management of HBV for HCWs might reduce the prevalence of infective HCWs and consequently the probability of HBV nosocomial infection from HCWs to their patients. Prevalence of HBV vaccination was low $(9.1 \%)$ while prevalence of exposure $(47.3 \%)$ and susceptibility $(43.5 \%)$ to HBV were high. There is thus a high need for sensitisation of HCWs in this area on HBV transmission and prevention. The sensitisation along with an effective and massive vaccination campaign should be carried out in this region not only among HCWs but in the population in general.

Acknowledgements All HCWs in the Bamenda Health District who consented to participate in this study; Dr Akassong D. Dinga Wonanke for helping with the purchase of material, Mr Fondoh Victor for his help at the start of this work, RHB for permitting the blood samples to be stored in the RHB laboratory freezer during sample collection.

Contributors EA and CT designed the study; EA and RN performed the experiments; EA drafted the manuscript; CT, RN, LA, J-RK and SK were involved in editing the manuscript; EA and SK performed the statistical analysis. All authors read and approved the final manuscript.

Funding The authors have not declared a specific grant for this research from any funding agency in the public, commercial or not-for-profit sectors.

Competing interests None declared.

Patient and public involvement Patients and/or the public were not involved in the design, or conduct, or reporting, or dissemination plans of this research.

\section{Patient consent for publication Not required.}

Ethics approval Ethical clearance for the study was obtained from the National Ethics Committee of Cameroon (N²017/02/871/CE/CNERSH/SP). Authorisation to carry out research in the NWR was obtained from the regional delegation. Authorisations to access different hospitals were obtained from the directors or the in-charge of the hospitals. Authorisation to access health centers was obtained from the district medical officer and the chief of centers of the health facilities in this region. Written informed consent was obtained from each participant.

Provenance and peer review Not commissioned; externally peer reviewed.

Data availability statement Data are available in a public, open access repository (https://doi.org/10.6084/m9.figshare.13503231.v1).

Open access This is an open access article distributed in accordance with the Creative Commons Attribution Non Commercial (CC BY-NC 4.0) license, which permits others to distribute, remix, adapt, build upon this work non-commercially, and license their derivative works on different terms, provided the original work is properly cited, appropriate credit is given, any changes made indicated, and the use is non-commercial. See: http://creativecommons.org/licenses/by-nc/4.0/.

ORCID iDs

Etheline W Akazong http://orcid.org/0000-0001-5389-2768

Richard Njouom http://orcid.org/0000-0003-3112-6370

\section{REFERENCES}

1 Kathleen D. Hepatitis B: causes, symptoms and treatment. Medical News Today, 2018.

2 World Health Organisation. Updated factsheet: hepatitis B. Geneva: WHO, 2020.

3 Njouom R, Tejiokem M, Texier G. Fontanet A. O-02: prevalence of hepatitis $B$, hepatitis $C$ and hepatitis $D$ virus infections in Cameroon: results from a national population based survey (the ANRS 12289 project). J Viral Hepat 2015;22:1-2.

4 World Health Organization. Myths and facts about immunization. WHO Regional Office for Europe, 2015.

5 WHO. Weekly epidemiological record. Geneva: WHO, 2009: 405-20.

6 Gatchalian S, Reyes M, Bernal N, et al. A new DTPw-HBV/Hib vaccine is immunogenic and safe when administered according to the EPI (expanded programme for immunization) schedule and following hepatitis $B$ vaccination at birth. Hum Vaccin 2005;1:198-203.

7 van der Sande MAB, Waight $\mathrm{P}$, Mendy $\mathrm{M}$, et al. Long-term protection against carriage of hepatitis $B$ virus after infant vaccination. $J$ Infect Dis 2006;193:1528-35.

8 World Health Organisation. Health care worker safety. In Geneva: WHO, 2003.

9 Jessica D, Kyle B, Costi D. Hepatitis B in health care workers: transmission events and guidance management. World $\mathrm{J}$ Hepat 2015;7:488-97.

10 Bilounga Ndongo C, Eteki L, Siedner M, et al. Prevalence and vaccination coverage of hepatitis $B$ among healthcare workers in Cameroon: a national seroprevalence survey. $J$ Viral Hepat 2018;25:1582-7.

11 Akazong W E, Tume C, Njouom R, et al. Knowledge, attitude and prevalence of hepatitis $B$ virus among healthcare workers: a crosssectional, hospital-based study in Bamenda Health District, NWR, Cameroon. BMJ Open 2020;10:e031075.

12 Tatsilong HOP, Noubiap JJN, Nansseu JRN, et al. Hepatitis $B$ infection awareness, vaccine perceptions and uptake, and serological profile of a group of health care workers in Yaoundé, Cameroon. BMC Public Health 2016;16.

13 Qin Y-L, Li B, Zhou Y-S, et al. Prevalence and associated knowledge of hepatitis $B$ infection among healthcare workers in Freetown, Sierra Leone. BMC Infect Dis 2018;18:315.

14 Smith SM. Determining-sample-size. Qualtrics, 2013.

15 Tandi TE, Cho Y, Akam AJ-C, et al. Cameroon public health sector: shortage and inequalities in geographic distribution of health personnel. Int J Equity Health 2015;14:43.

16 Mueller A, Stoetter L, Kalluvya S, et al. Prevalence of hepatitis B virus infection among health care workers in a tertiary hospital in Tanzania. BMC Infect Dis 2015;15:386.

17 Fritzsche C, Becker F, Hemmer CJ, et al. Hepatitis B and C: neglected diseases among health care workers in Cameroon. Trans $R$ Soc Trop Med Hyg 2013;107:158-64.

18 Loriette M, Birguel J, Damza R, et al. An experience of hepatitis B control in a rural area in far North Cameroon. Médecine et Santé Tropicales 2015;25:422-7. 
19 Erhabor O, Kwaifa I, Bayawa A. Comparison of ELISA and rapid screening techniques for the detection of HBsAg among blood donors in Usmanu Danfodiyo University teaching hospital Sokoto, North Western Nigeria [Internet]. J Blood \& Lymph 2014.

20 Hayder I, Ahmed W, Alam SE. Comparison of different ICT kits for HBsAg and anti HCV using gold standard ELISA. Pak J Med Res 2012;51:72-6.

21 Cruz HM, Scalioni LdeP, Paula VSde, et al. Poor sensitivity of rapid tests for the detection of antibodies to the hepatitis $\mathrm{B}$ virus: implications for field studies. Mem Inst Oswaldo Cruz 2017;112:209-13.

22 Cruz HM, Scalioni LdeP, de Paula VS, et al. Evaluating HBsAg rapid test performance for different biological samples from low and high infection rate settings \& populations. BMC Infect Dis 2015;15:548-58.

23 Ott JJ, Stevens GA, Groeger J, et al. Global epidemiology of hepatitis B virus infection: new estimates of age-specific $\mathrm{HBsAg}$ seroprevalence and endemicity. Vaccine 2012;30:2212-9.
24 Fattovich G, Zagni I, Scattolini C. Natural history of Hepatitis B and prognostic factors of disease progression. In: Marcellin P, ed. Management of patients with viral hepatitis. Paris, 2004: 203-20.

25 Ortona E, Pierdominici M, Rider V. Editorial: sex hormones and gender differences in immune responses. Front Immunol 2019;10:1076.

26 World Health Organisation. Training modules on hepatitis $B$ and $C$ screening, diagnosis and treatment-session 8. Geneva, 2020.

27 Villar LM, Cruz HM, Barbosa JR, et al. Update on hepatitis B and C virus diagnosis. World J Virol 2015;4:323.

28 World Health Organization, Global Hepatitis Programme. Guidelines for the prevention, care, and treatment of persons with chronic hepatitis B infection [online]. Geneva: WHO, 2015. http://apps.who. int/iris/bitstream/10665/154590/1/9789241549059_eng.pdf?ua=1\& ua $=1$

29 Centers for Disease Control and Prevention. CDC updates HBV vaccination guidelines for HCWs [online], 2014. Available: https:// www.clinicaladvisor.com/web-exclusives/cdc-updates-hbvvaccination-guidelines-for-hcws/article/327757/\# [Accessed 11 Dec 2018]. 